\title{
Simulation-Based Climbing Capability Analysis for Quadrupedal Robots
}

\section{Conference Paper}

\section{Author(s):}

Uno, Kentaro; Valsecchi, Giorgio; Hutter, Marco (ID); Yoshida, Kazuya

Publication date:

2021

Permanent link:

https://doi.org/10.3929/ethz-b-000501538

Rights / license:

In Copyright - Non-Commercial Use Permitted

Originally published in:

Lecture Notes in Networks and Systems 324, https://doi.org/10.1007/978-3-030-86294-7_16 


\title{
Simulation-Based Climbing Capability Analysis for Quadrupedal Robots
}

\author{
Kentaro Uno ${ }^{*}$, Giorgio Valsecchi ${ }^{2} *$, Marco Hutter $^{2}$, and Kazuya Yoshida ${ }^{1}$ \\ 1 Space Robotics Lab (SRL), Dep. of Aerospace Engineering, Tohoku University \\ unoken@dc.tohoku.ac.jp, yoshida.astro@tohoku.ac.jp \\ 2 Robotic Systems Lab (RSL), ETH Zurich \\ vgiorgio@ethz.ch, mahutter@ethz.ch
}

\begin{abstract}
The design of legged robotic systems targeted for climbing on steep terrain is critical for expanding robotic exploration in challenging terrain. While the most advanced quadruped robots show encouraging performance in level locomotion, there is little knowledge on the optimal design for climbing legged robots. In this paper, we investigate the climbing performance of quadrupedal systems with different joint topologies. To this end, we present a quantitative comparison performed in simulations of two robots in different configurations concerning locomotion stability, energy efficiency, and control versatility. Based on the results, optimized nominal stances are selected for each robot, and their climbing locomotion is demonstrated and compared in a virtual deployment.
\end{abstract}

Keywords: Legged Robots, Climbing Robots, Simulation Analysis, Topology Design, Robot Stability

\section{Introduction}

Climbing robots have the potential to significantly expand the exploration capabilities of robots on extraterrestrial bodies, where total robot mass and available energy are critically limited. To expand the exploration coverage in such environments with possibly steep inclinations, it is crucial to design the leg's topology to maximize the climbing capabilities. To this end, the number of legs, their number of joints, and their orientation should be optimized.

To date, legged systems are often designed with inspiration from the biological counterpart, which leads to body and leg structures similar to animals and humans. In nature, different species of mammals and arthropods can travel up and down steep and complex terrains. Examples include an alpine ibex shown in Fig. 1(a) and a harvestman in Fig. 1(b). They have different limb configurations; the mammal has legs opened back and forth two by two, and the spider looks suspending its torsor from the knee joints. Although both animals show a potential to climb, a fair comparison of the two types of limb configuration seems hard due to the big difference in mass and the body structural dimension - and it is unclear how these results would transfer to robotics counterparts.

* These authors contributed equally to this work. 


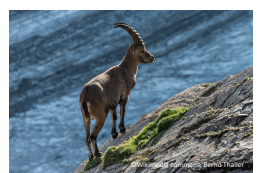

(a) Alpine ibex

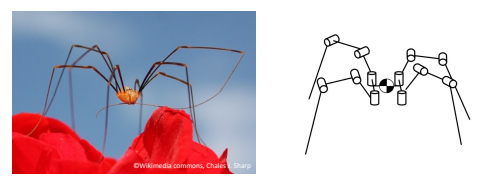

(b) Harvestman

Fig. 1: Mammalian and insect species compared to their topological representations as 12 DOF four-legged robotic systems.

Surveying the real walking robots' limb design, four legs with three actuated degrees of freedom (DOF) is the most frequently used solution, which is the minimum setup to execute statically stable walking gaits. There exist two major joints configurations to exploit three DOFs, called mammal and insect (spider) configurations. Ordering three rotational joints from the robot base side, only the first rotational direction is different between the two configurations. In the mammal configuration, the first joint axis is parallel to the front-facing direction, whereas the insect configuration's first joint axis is vertical. In both configurations, the second and third joints have horizontal axes perpendicular to the first joint.

In this work, we examine the climbing capability of the mammalian and insect robots with the same link dimension and inertial property in terms of stability, efficiency, and manipulability.

\section{$1.1 \quad$ Related Work}

The optimal topology of walking and climbing robots' legs has already been investigated from a high-level perspective. For example, [1] introduced numerical approach and well-defined metrics, and [2], [3] partially considered the case of climbing robots.

With the rise of computational power and new optimization and learning methods, there is a trend towards applying computational tools to synthesize, optimize, and compare different designs. To mention just a few: [4] jointly optimized the walking gait and the morphology, [5] optimized the limbs of a walking robot for specific tasks, and [6] proposed a framework for optimizing the topology of robotic legs for specific locomotion targets.

In addition to design, other works conducted with state-of-the-art climbing robots, such as LEMUR 3 [7], Magneto, [8], and HubRobo [9] with the passive spine gripper [10] focus more on the integration and control aspects.

Despite the progress in the field, there is still no preferred topology for climbing robots. It is enough to look at the already mention robots and few others, like Capuchin [11], Lemur IIa [12], IIb [13], Spaceclimber [14], to realize how different design can be. These robots differ for leg numbers, actuated and passive joints numbers, joints direction, and range of motion. Moreover, their different technological readiness level and testing make hard to compare their performances and draw general conclusions about the ideal topology. 


\subsection{Contribution}

This work aims at partially filling the knowledge gap regarding the optimal design of climbing robots, with a specific focus on legged robots. For this reason, we perform a simulation-based evaluation of quadruped robots with the two different joint configurations in the limbs, the aforementioned insect and mammalian, focusing on climbing scenarios. Configurations with a different number of legs have not been considered because they would hardly be comparable among each other with the proposed metrics. Other types of robot, such as the wheeled robot, have not been considered because incompatible with the free climbing motion that is the object of this study. The proposed analysis offers some insights on the effects of the legs' topology, which could guide future designs.

In section 2 we introduce the metrics and the robot models used for the analysis, together with the simulation environment. In section 3 , firstly we compare different nominal stance configurations. Then a static simulation, assuming the robots are holding on a steeply inclined slope, is performed. The behavior of the stability and the joint torque consumption across the workspace are analyzed. A dynamic climbing simulation, assuming realistic climbing gaits, is then used to compare the locomotion performance. Finally, in section 4 we provide an interpretation for the tests' outcome.

\section{Method}

\subsection{Multiaspect Evaluation}

Making a comprehensive comparison between two different robot topologies is difficult because of the lack of an absolute metric. For this reason, the comparison is conducted by using various metrics, focusing on different aspects of locomotion. In particular, we aim at assessing the capabilities of a legged robot in terms of stability, efficiency, and versatility. These parameters have been selected because directly affected by the topology and while having a strong influence on the actual mission and quality of the performance.

As a quantitative criterion of stability for climbing motion, we employ Tumble Stability Margin (TSM) [15] and Gravito-Inertial Acceleration Margin (GIAM) [16]. TSM is a generalization of the stability margin for climbing robots.

TSM is calculated based on the fact that the legged robot's tumbling necessarily passes through the state of two supporting points. The tumbling moment $M_{a b}$ acting around the arbitrary support line between the two feet position $\boldsymbol{p}_{a}$ and $\boldsymbol{p}_{b}$ is calculated from the resultant moment $\overline{\boldsymbol{M}}$ and the resultant force $\overline{\boldsymbol{F}}$ acting on the robot as shown in Eq. (1).

$$
M_{a b}=\overline{\boldsymbol{M}} \cdot \frac{\left(\boldsymbol{p}_{a}-\boldsymbol{p}_{b}\right)}{\left|\boldsymbol{p}_{a}-\boldsymbol{p}_{b}\right|}+\overline{\boldsymbol{F}} \cdot \frac{\left(\boldsymbol{p}_{b} \times \boldsymbol{p}_{a}\right)}{\left|\boldsymbol{p}_{a}-\boldsymbol{p}_{b}\right|}
$$

The robot is in equilibrium on the support line $a-b$ if $M_{a b}$ is null or is in a direction that is supported by another contact point. If this condition is satisfied 
for all possible support lines, the robot is in equilibrium [15]. Simultaneously TSM is calculated as the minimum of $M_{a b}$ acting to the stable direction divided by the weight of the robot as in the following Eq. (2).

$$
T S M=\frac{\min \left|M_{a b}\right|}{m g}
$$

[17] introduced a technique to include the gripping force effect into the calculation of the total tumbling moment. This analysis relies on the same method.

On the other hand, GIAM is also derived based on the tumbling moment. By reforming the total tumbling moment equation in terms of the possible limitation of the GIA vector $\boldsymbol{a}_{g i}^{\lim }$, Eq. (3) is obtained.

$$
m \boldsymbol{a}_{g i}^{\lim } \cdot \boldsymbol{n}_{g a b}=\sum_{j} \boldsymbol{F}_{j} \cdot\left(\boldsymbol{p}_{b}-\boldsymbol{p}_{j}\right) \times\left(\boldsymbol{p}_{a}-\boldsymbol{p}_{j}\right)+\boldsymbol{M}_{0} \cdot\left(\boldsymbol{p}_{a}-\boldsymbol{p}_{b}\right)+\boldsymbol{F}_{0} \cdot\left(\boldsymbol{p}_{b} \times \boldsymbol{p}_{a}\right)
$$

$\boldsymbol{p}_{j}$ is the supporting position where the gripping force of $\boldsymbol{F}_{j}$ acts, $\boldsymbol{M}_{0}$ and $\boldsymbol{F}_{0}$ are external components of the tumbling moment. Eq. (3) is actually the expression of the equation of a three-dimensional plane in terms of GIA, dividing the acceleration space into stable and unstable region, where the vector $\boldsymbol{n}_{g a b}=\left(\boldsymbol{p}_{g}-\boldsymbol{p}_{a}\right) \times\left(\boldsymbol{p}_{g}-\boldsymbol{p}_{b}\right)$ is perpendicular to the tumbling axis $a-b$ from the center of gravity. If the GIA escapes from the stable side to unstable side, the robot tumbles over. GIAM is defined as the addable acceleration magnitude for GIA not to reach to the plane described in Eq. (4) [16].

$$
a_{\text {marg }}=\min \left(\left|\boldsymbol{a}_{g i, \lim }\right|-\frac{\boldsymbol{n}_{g a b} \cdot \boldsymbol{a}_{g i}}{\left|\boldsymbol{n}_{g a b}\right|}\right)
$$

It is important to state that the adhesion effect is considered as $\boldsymbol{F}_{j}$ in Eq. (3), indicating the gripper is effective to increase the stability margin.

Efficiency is another important aspect to evaluate. We measure torques acting at all the joints, using the maximum and root mean square (RMS) metrics. The RMS value correlates quadratically with the power consumption of the actuators. At the same time, the maximum torque gives information about how stressed are the single actuators and how uneven the load is.

Robustness and versatility are also essential components of the performance of a robot. To measure it, we consider the robot capability of applying forces in arbitrary directions while being in a specific pose. This property is compared using a standard manipulability measure.

The insights from the different metrics are then summarized and interpreted in the conclusion paragraph.

\subsection{Robot model}

A rigid-body model of the robots is necessary to perform the simulations necessary for the comparison. The selected models should be as sufficiently similar in size to guarantee a fair comparison while being equally good instances of the 


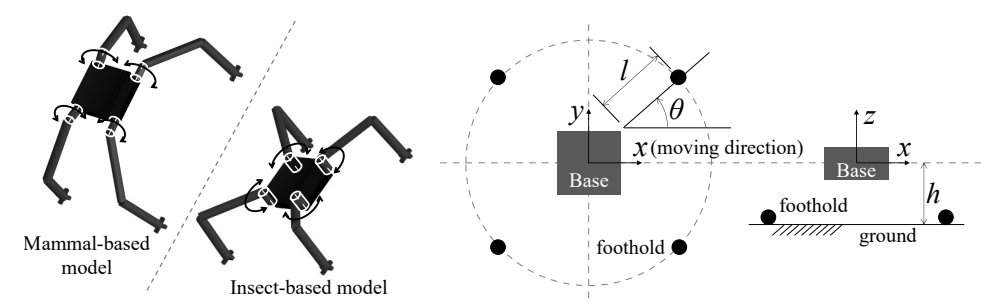

Fig. 2: Used robot models and three parameters to fix the nominal stance. Limbs configurations are symmetrical about $x$ and $y$ axes.

respective topologies and at the same time be also representative of realistic robots.

For this reason, a first guess of the design was made starting from the available data from ANYmal [18], to estimate the inertia and geometry of the bodies. The framework Vitruvio [6] was used to optimize the link lengths and validate the models. Robotic models for this work are shown in Fig. 2. The mammal-based model has been turned into an insect by simply rotating the leg's first joint to obtain a vertical axis. All the link lengths are maintained equally. Finally, a maximum gripping force of $200 \mathrm{~N}$ was fixed for both the robots, assuming that the gripping performance would be independent of the configuration and scaling values from other climbing robots.

Both robots model have a total mass of $28.3 \mathrm{~kg}$ and legs with a total reach of $0.85 \mathrm{~m}$, links have very similar lengths. The legs are all equal for simplicity and to preserve omnidirectionality.

\subsection{Simulation Setup}

For the result's repeatability and reproducibility, we employed ClimbLab [19] as a simulation platform dedicatedly designed for legged climbing robots and already open-sourced. In the simulations, the robot featured in the previous section is assumed to climb up the slope inclined by $45^{\circ}$ in the Earth gravity, which usually is tough to traverse for the conventional wheeled and tracked vehicle.

For this simulation analysis, the nominal stance is defined with the following parameters as shown in Fig. 2: distance from the corner of the base to the feet $l$, angle of external rotation from the moving direction $\theta$, and the base height from the ground surface $h$. For the robot model introduced above, $l$ of $0.45 \mathrm{~m}$ is selected to gain a suitable workspace.

\section{Results}

\subsection{Nominal Stance Analysis}

We performed simple dynamic simulations where the robot climbed on the slope with the standard gait with different $\theta$ from $0^{\circ}$ to $90^{\circ}$ to investigate the general 
characteristic by leg spreading. In this gait, the base height is kept constant $0.20 \mathrm{~m}$, and each limb swing trajectory is set as the same spline curves satisfying lifting and landing normal velocities are zero with the constant stride and swing height. It should be noted that the used gait is simple, and it does not actively optimize forces at the grippers. The gait period is set $8 \mathrm{~s}$ thus, each swing period $2 \mathrm{~s}$. All climbing simulations are run for two periods with GIAM and measure of manipulability recorded.

As shown in Fig. 3, robots of both topologies shows similar characteristics of GIAM along with the change of $\theta$. This is understandable because there cannot be a significant difference in inertial force distribution of the links between the two topologies when the gait speed is slow. Though GIAM can be improved by advanced motion control for both topologies, the characteristics are expected still similar if the gait policy is the same. On the other hand, a decrease in the mammal configuration's manipulability is observed in case $\theta$ is small and legs are not spread very much. In contrast, the insect configuration is robust in manipulability against the change of $\theta$. Here, it is important to be noted that the manipulability measure for both 3 DOFs manipulators is independent of the first joint angular change. The insect robot's manipulability is not affected because the configuration does not require the second and third joint adjustment along with the change of $\theta$.
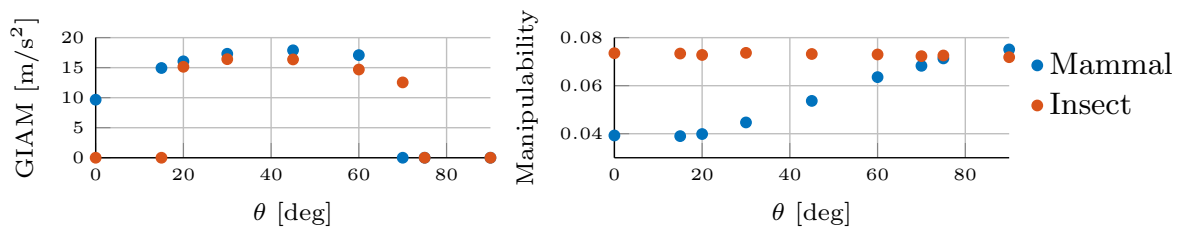

Fig. 3: Similar characteristic of GIAM and different response of manipulability against the change of $\theta$ for the two topologies. All plots are averaged values through each climbing. Zero of GIAM represents the case that the robot tipped over.

Another effective technique to increase slope climbing stability is lowering the base close to the ground since it minimizes the face-up pitching moment generated by the weight. That stance, however, requires the legs to be folded, which causes the decrease of manipulability. This technique's GIAM improvement is observed in the simulation result for both typed robots as displayed in Fig. 4. However, there is a significant difference in manipulability. While the mammal robot should sacrifice the manipulability to gain the lowered base stance, the insect robot can maintain almost the same manipulability and simultaneously adjust the base position.

Eventually, the insect robot can adjust the base pose without changing the foothold positions without affecting the manipulability. 

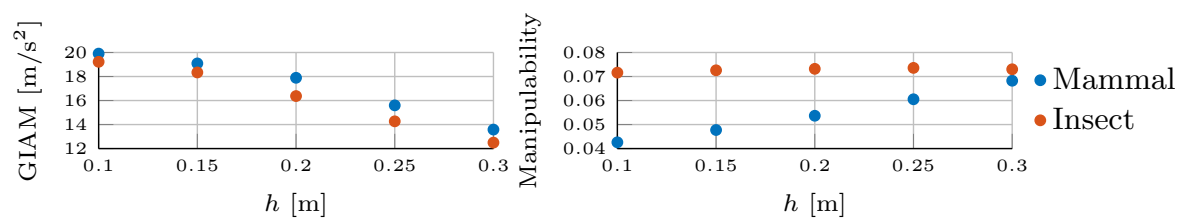

Fig. 4: Similar characteristic of GIAM and different response of manipulability against the change of the base height for the two topologies. All plots are averaged values through each climbing. Insect typed robot can lower the base to improve the stability without sacrificing the manipulability.

\subsection{Static Analysis}

To evaluate more in detail the performance of the two topologies, we performed a static analysis. In this analysis, footholds are fixed on a $45^{\circ}$ tilted slope, and the base is moved across the workspace. Then the metrics are recorded at the specific stance, in stationary conditions. These static measurements are iterated with a spatial resolution of $1.5 \mathrm{~cm}$. As for the nominal stance, $\theta=45^{\circ}$ is selected according to the previous analysis result.
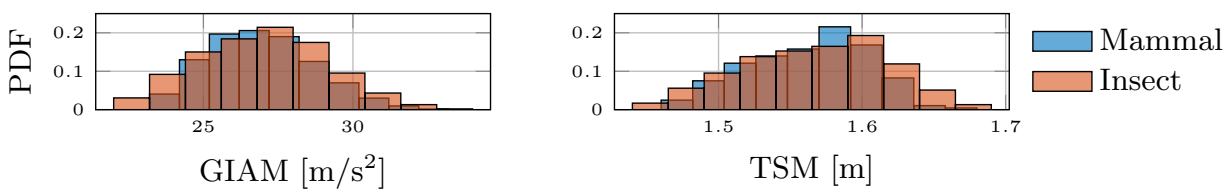

Fig. 5: Probability density function for the GIAM and TSM metrics, when measured across the whole workspace.

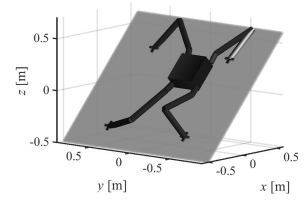

(a) Mammal

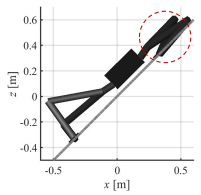

$x[m]$
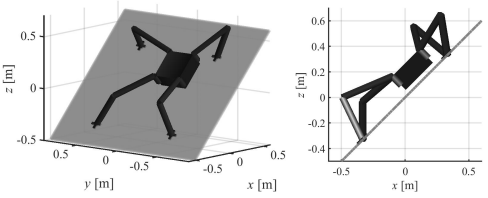

(b) Insect

Fig. 6: Different ground clearance of the limbs at lower base height for the two topologies. The inclined gray surface/line represents the ground. On the top right area, the insect's legs point away from the ground while the mammal interfered. 

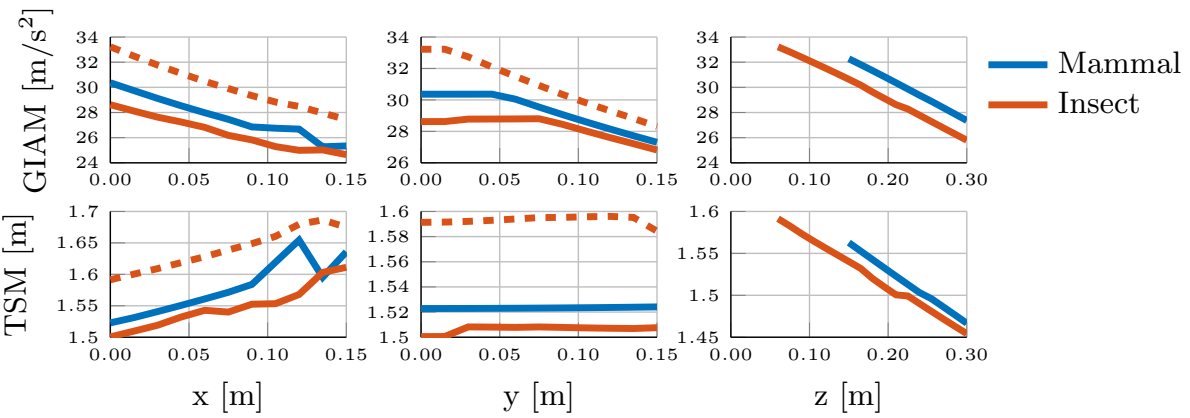

Fig. 7: TSM and GIAM metrics across the workspace for the two robot configurations. Continuous lines are for a base height of $0.2 \mathrm{~m}$ and dashed lines are for a base height of $0.06 \mathrm{~m}$, achievable only by the insect configuration.

Stability: TSM and GIAM, introduced in section 2, yield very similar values for the two robot configurations when measured across the whole workspace. Histograms illustrate this in Fig. 5. It can be explained by looking at the definitions of the metrics, which are primarily influenced by the footholds' and center of mass positions.

However, the insect configuration can lower the base more without having its limbs conflicting with the ground, as shown in Fig. 6. The better placement of the CoM thus increases the GIAM and the TSM.

Fig. 7 shows how the stability metrics vary across the workspace. It is evident how the capability of the insect configuration of lowering its center of mass more (plots on the right, for the $z$ coordinate) allows achieving higher values for the stability metrics. The four plots on the left ( $x$ and $y$ coordinates) confirm that the lower base effect is also stable across the workspace. It must be noted that improvement of the stability metrics due to the base height is only marginal, $5 \%$ to $15 \%$, but not negligible.
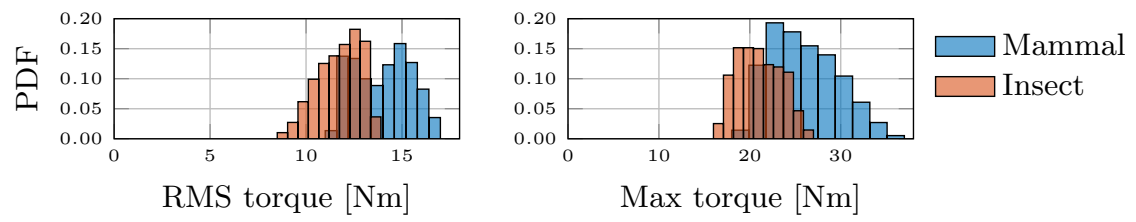

Fig. 8: Probability density function for the RMS and maximum torques, when measured across the whole workspace. The $x$ axis starts from zero intentionally to illustrate the relevance of the difference between the distribution. 


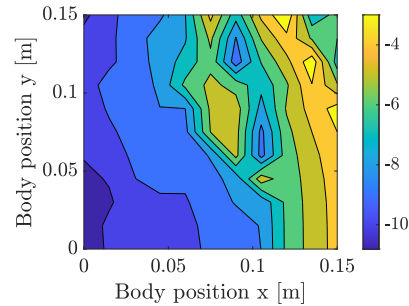

(a) RMS torque

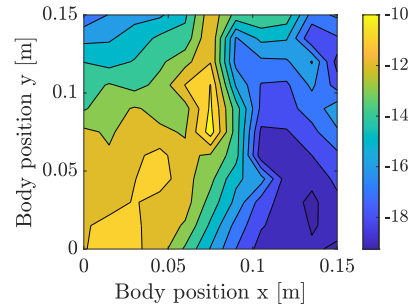

(b) Max torque

Fig. 9: Torque reduction, as a percentage, of the insect configuration when compared to the mammal. Measurements were taken on $x y$-plane, with a base height of $0.06 \mathrm{~m}$ and $0.15 \mathrm{~m}$ for the insect and the mammal configuration, respectively.

Torque and energy efficiency: The histograms in Fig. 8 show the joints' torque distribution across the two robots' whole workspace. The difference in both cases is comparable to the variance; this means it is statistically significant. Moreover, also the highest maximum torque value is lower for the insect, making the extreme configuration less challenging from a design point of view.

The heat-maps in Fig. 9 show how much lower the torque is for the insect configuration, as a percentage when a single $x y$-plane is considered. In particular, the lowest planes achievable by the robot were used. This plot demonstrates how the different distributions of Fig. 8 are not a consequence of a different spatial distribution of torque; they represent instead consistently lower torques across the workspace.

While the torque reduction is only moderate, $10 \%$ to $20 \%$, it bears important consequences for the robots' overall design and capabilities. In particular, the actuators' winding losses (which account for a significant component of the total energetic consumption) scale quadratically to the torque, meaning that the insect configuration can expect a $20 \%$ to $40 \%$ lower heat generation from winding losses. Overall, the insect has a better torque to weight ratio for this use-case, fewer problems with heat dissipation, and should allow for more optimized designs.

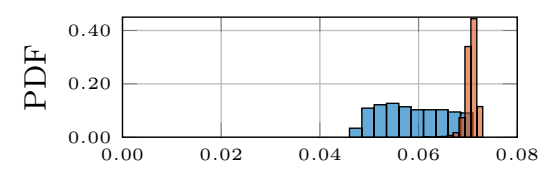

Mean manipulability measure

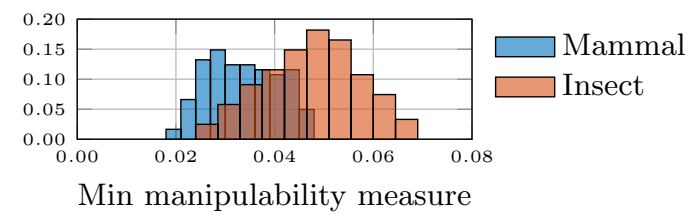

Min manipulability measure

Fig. 10: Probability density function of the manipulability measure across the whole workspace for the two robot configurations. 


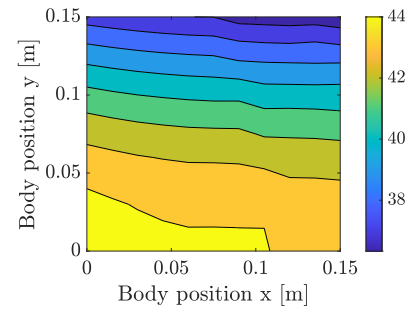

(a) Mean manipulability

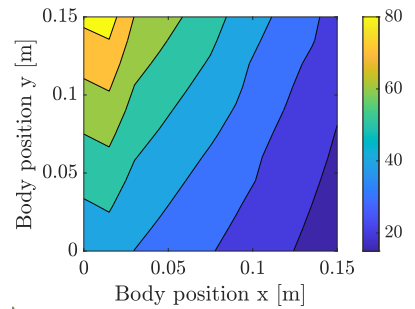

(b) Min manipulability

Fig. 11: Manipulability measure increase, as a percentage, of the insect configuration with respect to the mammal configuration on a $x y$-plane. Base height of $0.06 \mathrm{~m}$ and $0.15 \mathrm{~m}$ for the insect and for the mammal configuration respectively.

Versatility: The histograms in Fig. 10 show the mean and the minimum manipulability of the robots' four limbs across the whole workspace. In this case, the distributions are completely different, with the insect configuration showing a much smaller variance.

The heat-maps in Fig. 11 show how much greater the manipulability measure is for the insect configuration, as a percentage when a single $x y$-plane is considered. Again, the lowest possible planes have been used for the comparison, in their most stable configuration from the climbing point of view. In these conditions, the insect shows a significantly higher, 40\%, manipulability measure. This means that the mammal configuration limbs are closer to a singularity and cannot produce forces in an arbitrary direction as effectively.

\subsection{Dynamic Climbing Analysis}

For a more practical climbing gait, each topology's nominal pose is updated as follows. The lowest possible base positions are respectively selected to maximize stability. As for the leg spreading angle for the mammal configuration, $\theta=60^{\circ}$ is chosen to avoid the significant decrease of manipulability based on the result in Fig. 3, while $\theta=45^{\circ}$ is selected for the insect type. In this simulation, the base position was controlled to keep a constant height from the ground and maintain the COM horizontal projection be at the crossing point of the two diagonal support lines so that the static stability is satisfied. Also, each swing duration was set $8 \mathrm{~s}$.

Fig. 12 shows the uphill climbing behavior of two topologies with the stabilityaware stance. In addition to the case of ascending, lateral walking was also simulated. Each metric while these two simulations are shown in Fig. 13. We can observe that the two robots are similarly stable according to TSM. However, the insect robot's manipulability is greater than the mammal in average. Furthermore, the insect robot requires relatively lower torques than the mammal robot. 

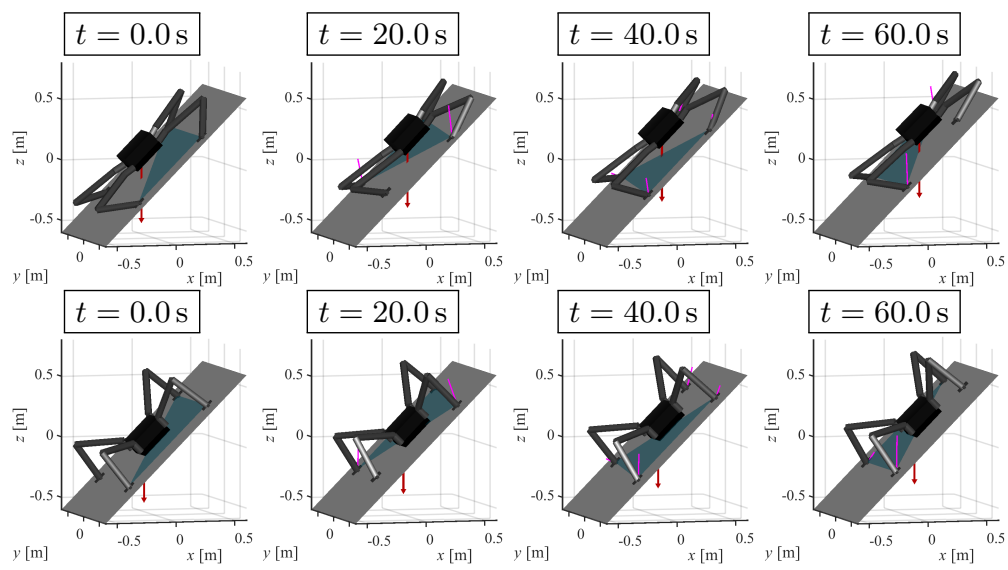

Fig. 12: Robot behaviors while uphill climbing on $45^{\circ}$ slope by the mammal robot (top row) and insect robot (bottom row) with final stability-aware stance.
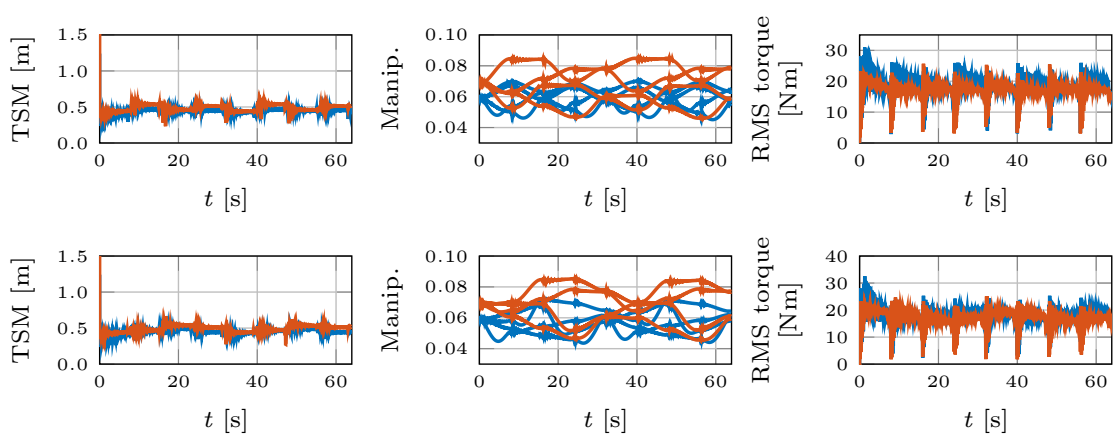

Fig. 13: Metrics recorded while uphill climbing (top row) and lateral walking (bottom row) on $45^{\circ}$ slope by the two robots with the stability-aware stance. While TSM showed very similar stability performances, the insect topology sustains higher manipulability in average and relatively less torque consumption.

\section{Conclusion}

In this paper, to quantitatively clarify quadruped robots' potential for climbing applications, we evaluated the two most common leg topologies, mammalian, and insect configurations, regarding stability, energy efficiency, and versatility. Both dynamic and static simulation results proved that the insect robot's capability to adjust the base pose without compromising the limb's manipulability, which in turn has a positive effect on stability. This higher manipulability of the insect robot's legs should grant more robustness during climbing in uneven terrains, Allowing the robot to react better to disturbances. Furthermore, torque consumption analysis showed the insect robot has higher energy efficiency, which 
means more payload and longer operational time with the identical actuators. In particular, the lower torque consumption holds when the robots are in their most stable configurations. In summary, the insect configuration results in being more stable, energy-efficient, and versatile at once.

While this work gives a hint for the robot hardware design towards climbing application, the software side approach could also improve the stability and efficiency of climbing locomotion, which will be addressed with the experimental validation as the authors' future work.

\section{References}

1. A. M. Abate, Mechanical design for robot locomotion, $\mathrm{PhD}$ thesis, Oregon State University, 2018.

2. S. Hirose and K. Arikawa, Advanced Robotics 15, 125 (2001).

3. S. Hirose et al., IEEE Robotics \& Automation Magazine 16, 104 (2009).

4. K. M. Digumarti et al., Concurrent optimization of mechanical design and locomotion control of a legged robot, in Mobile Service Robotics, 2014 pp. 315-323.

5. S. Ha et al., Task-based limb optimization for legged robots, in Proc. IEEE/RSJ Int. Conf. Intell. Rob. and Syst., 2016.

6. M. Chadwick et al., IEEE Robot. Automat. Lett. 5, 6318 (2020).

7. A. Parness et al., Lemur 3: A limbed climbing robot for extreme terrain mobility in space, in Proc. IEEE Int. Conf. Robot. Automat., 2017.

8. T. Bandyopadhyay et al., Magneto: A versatile multi-limbed inspection robot, in Proc. IEEE/RSJ Int. Conf. Intell. Rob. Syst., 2018.

9. K. Uno et al., Hubrobo: A lightweight multi-limbed climbing robot for exploration in challenging terrain, in Proc. IEEE-RAS Int. Conf. Human. Rob., 2021.

10. K. Nagaoka et al., IEEE Robot. Automat. Lett. 3, 1765 (2018).

11. R. Zhang and J.-C. Latombe, Int. J. Advanced Robotic Systems 10, p. 194 (2013).

12. K. Nickels et al., Lemur iia capabilities, in Proc. IEEE/RSJ Int. Conf. Intell. Rob. Syst., 2006.

13. J. Dietsch et al., Industrial Robot: An Int. J. (2006).

14. S. Bartsch, KI-Künstliche Intelligenz 28, 127 (2014).

15. K. Yoneda and S. Hirose, Tumble stability criterion of integrated locomotion and manipulation, in Proc. IEEE/RSJ Int. Conf. Intell. Rob. Syst., 1996.

16. W. F. R. Ribeiro et al., Dynamic equilibrium of climbing robots based on stability polyhedron for gravito-inertial acceleration, in Proc. CLAWAR, 2020.

17. K. Uno et al., Gait planning for a free-climbing robot based on tumble stability, in Proc. IEEE/SICE Int. Symp. on Syst. Integrat., 2019.

18. M. Hutter et al., Anymal - a highly mobile and dynamic quadrupedal robot, in Proc. IEEE/RSJ Int. Conf. Intell. Rob. Syst., 2016.

19. K. Uno et al., Climblab: MATLAB simulation platform for legged climbing robotics, in Proc. CLAWAR, 2021.

\section{Acknowledgments}

This work was supported by JSPS KAKENHI Grant Number 19J20685 and ESA Contract Number 4000131516/20/NL/MH/ic. The authors also want to thank Jordis E. Herrmann for her valuable contributions. 